American Journal of Pharmaceutical Education 2018; 82 (9) Article 6424.

\title{
RESEARCH
}

\section{Pharmacogenomics Education Improves Pharmacy Student Perceptions of Their Abilities and Roles in Its Use}

\author{
Rebecca Marcinak, PharmD, Meaghan Paris, PharmD, Shannon R. M. Kinney, PhD \\ Western New England University, College of Pharmacy, Springfield, Massachusetts \\ Submitted March 24, 2017; accepted June 15, 2017; published November 2018.
}

Objective. To assess whether a required first-year course, Principles in Genetics and Pharmacogenomics, and integration into subsequent courses affected pharmacy students' perceptions of pharmacogenomics. Methods. A survey was distributed to Professional Year (PY) 1 students during the first and last weeks of the course from 2014 to 2016. A follow-up survey was distributed to PY2, PY3, and PY4 students.

Results. Respondents consistently agreed that pharmacogenomics is clinically relevant. After the course, PY1 students are more comfortable in their knowledge and role in the application of pharmacogenomics. Although their comfort reverts to some degree, PY2-PY4 students believe that they should be able to apply pharmacogenomics clinically. Most PY2-PY4 students indicate that later courses review pharmacogenomics.

Conclusion. A required course in genetics and pharmacogenomics can promote a perception that pharmacists should have knowledge of, and be involved in the use of genetic information clinically. Inclusion of pharmacogenomic concepts in subsequent curricular components may help in maintaining these perceptions.

Keywords: pharmacogenomics education, pharmacy

\section{INTRODUCTION}

Pharmacogenomics, the study of how genetics affect patient response to medications, has the potential to improve pharmacotherapy and to allow for a better understanding of diseases. ${ }^{1}$ In addition, it is thought that genetics may be a potential solution to the attrition of many drugs in late-stage clinical trials, as well as reduced efficacy or appearance of adverse effects once medications become available to the public. ${ }^{2}$ As the field of pharmacogenomics grows, there is an increasing need for clinicians with the knowledge and skills to determine therapy recommendations based on patient specific genetic information, termed personalized medicine. ${ }^{3}$ Pharmacists and pharmacy graduates fill a unique role, whether it is at a retail pharmacy, in pharmaceutical industry, governmental regulation or with an interdisciplinary team of health professionals where they can combine genetic information with their knowledge of drug pharmacokinetics and pharmacodynamics to provide individualized therapy for the best clinical outcome. However, in a 2012 publication, although

Corresponding Author: Shannon Kinney, Western New England University, Center for Sciences and Pharmacy, Room 322, 1215 Wilbraham Rd., Springfield, MA, 01119-2684. Tel: 413-796-2439. Fax: 413-796-2266. E-mail: Shannon.Kinney@, WNE.edu more than $90 \%$ of pharmacists were interested in learning more about pharmacogenomics, $83 \%$ of pharmacists rated their knowledge of pharmacogenomics as only "fair" or even "poor." Similarly, in a 2011 study, 63\% of pharmacists believed they could not accurately apply pharmacogenomics test results to drug therapy, selection and monitoring, though most $(85 \%)$ still agreed that pharmacists should be required to be knowledgeable about pharmacogenomics. ${ }^{5}$ Because the field of pharmacogenomics is relatively new, many current pharmacists have not received a substantial amount of education on this topic during their pharmacy degree training and a number of continuing education programs are making an effort to increase their knowledge. ${ }^{6,7}$ The other major component to solving this problem is through education of prospective pharmacists to ensure that these numbers shift in a positive direction in the future.

There are limited data available on the perceptions of pharmacy students on the topic of pharmacogenomics. Of the few studies that have been completed to date, one 2013 study indicated that a majority $(75.3 \%)$ of pharmacy students at the University of Minnesota (UMN) agreed that pharmacogenomics should be an important part of their curriculum, yet only $13.1 \%$ perceived that it was. ${ }^{8}$ Furthermore, approximately $90 \%$ of pharmacy students believed that they should be required to be 


\section{American Journal of Pharmaceutical Education 2018; 82 (9) Article 6424.}

knowledgeable about this subject and once in practice, they should be able to identify medications requiring genetic testing, which was a higher percentage than both medical and nursing students. ${ }^{8}$ Only approximately $13 \%$ of pharmacy students at UMN agreed that they knew which medications required pharmacogenomics testing. However, those that indicated that they had taken classes connecting genetics to pharmacy were more likely to agree that they were knowledgeable about the subject and comfortable with the application of pharmacogenomics. ${ }^{8}$ Interestingly, of the three fields of health care students surveyed, pharmacy students appeared to feel more responsible for being knowledgeable about pharmacogenomics. A second 2012 study surveying students across pharmacy programs in California reported that while almost all pharmacy students were aware of what pharmacogenomics was, and perceive that it will be important for future pharmacists, they were neutral in regards to pharmacists being prepared to have a role in pharmacogenomics. ${ }^{9}$

Since the early 2000s, several organizations, including the American Association of Colleges of Pharmacy (AACP), have recommended that pharmacy programs include education on the topic of pharmacogenomics in their curricula and have outlined competencies related to genetics that should be achieved by pharmacists. ${ }^{10}$ In an effort to further promote the inclusion of pharmacogenomics education in pharmacy programs, the latest accreditation standards ("Standards 2016") provided by the Accreditation Council for Pharmacy Education (ACPE), include pharmacogenomics as a required element of the didactic Doctor of Pharmacy Curriculum. ${ }^{11}$ Furthermore, ACPE emphasizes that this cannot be an elective component of the curriculum and should be taught at an appropriate level for students to retain and apply their knowledge to patient care in various practice settings." 11 Additionally, in the American Society of Health-System Pharmacists (ASHP) Statement on the Pharmacist's Role in Clinical pharmacogenomics, they encourage both pharmacogenomics education and use of genetics by pharmacists when making therapeutic decisions. ${ }^{12}$

Despite these recommendations, not all pharmacy programs currently offer courses in genetics and pharmacogenomics. A study conducted among U.S. pharmacy schools in 2010 , revealed that $89.3 \%$ of pharmacy schools offered pharmacogenomics in their PharmD curriculum. However, only $21.7 \%$ of PharmD curricula had a standalone required didactic course in the area. Further, only $56.5 \%$ of schools offered more than ten didactic hours on this topic and $53.3 \%$ of pharmacy programs named the present state of pharmacogenomics instruction at most schools of pharmacy as "poor". ${ }^{13}$ A 2016 study found that this had increased slightly, with $35 \%$ of pharmacy programs having a required standalone pharmacogenomics course in the curriculum, with new programs being more likely to have a required course than older programs. ${ }^{14}$ Although there has been growth in pharmacogenomics education, especially as new pharmacy programs are opening, there is still room for modification - and addition - of pharmacy education in this area. There is still the question of how much and what type of exposure to pharmacogenomics is effective for students to obtain the knowledge, skills and comfort required to apply these principles to patient care. Thus, data indicating the usefulness of such courses, as well as information on their objectives and layout, may be helpful in making curricular decisions on this topic.

At Western New England University (WNE), there is a required standalone didactic 2 credit hour course entitled Principles of Genetics and Pharmacogenomics during the Spring of PY1 year. The course learning objectives aim to provide proficiency in understanding the mechanisms by which genetic inheritance can lead to disease, understanding how genetic variations can affect basic pharmacokinetic and pharmacologic principles, predicting treatment regimens that may be influenced by an individual's genomic composition with use of case-based application and The Pharmacogenomics Knowledgebase (PharmGKB) and The Clinical Pharmacogenetics Implementation Consortium (CPIC) guidelines as resources, and weighing the benefits of using patient genetic information with the social and ethical consequences that may arise. In addition, all of the core competencies perceived to be important to US pharmacy schools and colleges that responded to a survey on pharmacogenomics and pharmacogenomics education in 2010 are covered. ${ }^{13}$ According to the above mentioned studies, the existence of a required course in pharmacogenomics occurs in about onethird of colleges of pharmacy across the country. ${ }^{14}$ Thus, in order to develop some perspective on the effectiveness of the course, as well as retention of the information after the course was completed, the authors investigated student perceptions of their knowledge and the use of genetic information in medication therapy while training in the program.

\section{METHODS}

Study participants were Doctor of Pharmacy students at Western New England University College of Pharmacy. Participants were introduced to pharmacogenomics during the spring semester of the first professional year in the required course, Principles of Genetics and Genomics. All surveys were reviewed and approved (exempted) by the Western New England University Institutional Review Board.

An online survey was designed to collect information on the perceptions of knowledge and of the importance 


\section{American Journal of Pharmaceutical Education 2018; 82 (9) Article 6424.}

of pharmacogenomics in the clinical setting of first year pharmacy students. Several of the survey questions were adapted from questions reported in earlier studies. ${ }^{8,15}$ Additional questions were developed by the authors. The survey link was distributed, via email, to PY1 (first professional year) PharmD students during the first week (presurvey) and after the last week (post-survey) of the required pharmacogenomics course. The pre-survey was issued to the first class of students in January 2014, the second in January 2015, and the third in January 2016. The post-survey was issued after the last course lecture of the same 3 years. The pre- and post-surveys were identical in content, and answers to questions were paired from the pre-survey to the post-survey, based on a code known only to the respondents, in order to assess changes in respondents' perceptions. The questionnaire included items that assess respondents' views on the relevance and importance of pharmacogenomics to clinical patient care settings, items on comfort level in interpreting and applying genetic information to individual patients in clinical practice, and a few demographic items (gender, ethnicity, age, educational background, and pharmacy experience). Students were given $0.5 \%$ bonus on their final grade for the course for each completed survey.

An analogous follow up online survey tool was designed to collect information on the inclusion of pharmacogenomics in later courses and experiential work, as well as student comfort level and perception of their abilities upon encountering the topic in other academic or clinical situations. The survey included many of the same demographic, perception, and comfort items as the PY1 questionnaire. The survey link was distributed in the 2015-2016 academic year via email to PY2, PY3, and PY4 students, who had completed at least three modules of the integrated therapeutic courses [renal, respiratory, and cardiovascular I, Integrated Patient Care and Patient Management (IPC\&PM) modules]. Therefore, they had all previously taken the required Principles of Genetics and Pharmacogenomics course up to three years previously. Students were contacted up to 3 times, with each date of contact being at least 6 weeks apart. No compensation was provided for PY2-4 students who completed the survey. Due to the timing of administration of the surveys, the PY2 and PY3 students had previously had the opportunity to participate in the pre/post-surveys, while the PY4 students were only able to participate in the follow up questionnaire. Items regarding which other courses in the PharmD curriculum included pharmacogenomics instruction (a list of 26 required courses and one elective fill in option was provided) and whether the respondents have encountered pharmacogenomics in clinical practice were included. Because respondents were asked to indicate which courses in the curriculum included pharmacogenomics content, but not all students had completed all courses, percentages were adjusted based on their place in the curriculum and the courses presented here were filtered to include those that were selected by at least $20 \%$ of respondents. The two surveys were 27 or 18 questions in length, respectively, and required approximately 5 to 10 minutes to complete.

All data collected were anonymous. Data were collected using the online survey software and questionnaire tool, SurveyMonkey. Answers to all Likert scale questions were transformed to numerical data (disagree $=1$, neither agree nor disagree $=2$, and agree $=3$ ). Statistical analyses and comparisons were performed utilizing Graphpad Prism Software (Version 6.0f, La Jolla, CA). Means and standard deviations were calculated for numerical data, which were then tested for normality through the D'Agostino \& Pearson omnibus normality test. Wilcoxon Rank Sum test (paired or unpaired depending on the questionnaire) was used to determine significant differences $(p<.05)$ between groups. A post hoc power analysis was completed using G*Power (Version 3.1 Dusseldorf, Germany) statistical software and found to be $93 \%$ for the pre/post survey and $95 \%$ for the follow-up survey, both with a relatively small effect size $(.25) .{ }^{16}$ Slight differences arose in the responses from the various groups of PY1-PY4 students. However, statistically there are no differences between the years when comparing similar data and therefore all PY1 responses were grouped together and PY2-PY4 responses together when determining if student perceptions changed over time.

\section{RESULTS}

The response rate for the PY1 pre- and post-surveys (completion of both surveys based on matching of a unique code) was $84 \%$ in 2014, 88\% in 2015, and 79\% in 2016. The response rate for the survey in second, third, and fourth year students was $61.8 \%$ in PY2, 55.4\% in PY3, and 64.3\% in PY4 groups. Table 1 provides a summary of demographic information among the respondents from the PY1 pre/post surveys. In all three PY1 groups, the majority of students were female $(57.1,69.2$, and $62.5 \%$ ), of younger age (17-24 years $82.6,86.4$, and $80.7 \%)$, white $(82.5$, 78.8 , and $75.4 \%$ ), had only completed pre-pharmacy/prerequisites $(73,78.8$, and $71.4 \%)$, and worked for a retail chain pharmacy $(71.4,56.1$, and $58.9 \%)$.

The WNE PharmD program is considered a $2 / 4$ professional program with most incoming students having either 2 years of pre-pharmacy education (at WNE, the pre-pharmacy curriculum does not include a genetics course) or a four year Bachelor of Science degree. The majority of the students had not taken a genetics course in the past, but learned some genetics in their basic science courses $(73,82$, and $68 \%$ ) (Table 1). There were some students $(3,3$, and $4 \%)$, however, who had never learned 
American Journal of Pharmaceutical Education 2018; 82 (9) Article 6424.

Table 1. Demographics and Genetics Education Background of PY1 Students

\begin{tabular}{|c|c|c|c|}
\hline & $\begin{array}{c}2014 \\
\%(n=74)\end{array}$ & $\begin{array}{c}2015 \\
\%(n=66)\end{array}$ & $\begin{array}{c}2016 \\
\%(n=57)\end{array}$ \\
\hline \multicolumn{4}{|l|}{ Gender } \\
\hline Male & 42.9 & 30.8 & 37.5 \\
\hline Female & 57.1 & 69.2 & 62.5 \\
\hline \multicolumn{4}{|l|}{ Age } \\
\hline $17-20$ & 41.3 & 36.4 & 36.8 \\
\hline $21-24$ & 41.3 & 50.0 & 43.9 \\
\hline $25-30$ & 15.9 & 10.6 & 15.8 \\
\hline $31-40$ & 1.6 & 3.0 & 3.5 \\
\hline \multicolumn{4}{|l|}{ Race } \\
\hline White & 82.5 & 78.8 & 75.4 \\
\hline Black or African-American & 6.3 & 1.5 & 10.5 \\
\hline Asian & 7.9 & 9.1 & 10.5 \\
\hline Hispanic & 1.6 & 1.5 & 1.8 \\
\hline Multiple races & 1.6 & 6.1 & 1.8 \\
\hline Other & & 3.0 & \\
\hline \multicolumn{4}{|l|}{ Highest level of education completed } \\
\hline Pre-pharmacy/prerequisites & 73 & 78.8 & 71.4 \\
\hline Bachelor's degree & 23.8 & 21.2 & 28.6 \\
\hline Master's degree & 1.6 & & \\
\hline Other & 1.6 & & \\
\hline \multicolumn{4}{|l|}{ Work experience in pharmacy } \\
\hline Retail chain & 71.4 & 56.1 & 58.9 \\
\hline Retail independent & 7.9 & 13.8 & 10.7 \\
\hline Hospital & 6.3 & 13.8 & 10.7 \\
\hline Ambulatory & 1.6 & 1.5 & \\
\hline Other (eg, industry) & 3.2 & 3.0 & 1.8 \\
\hline None & 9.5 & 12.1 & 17.9 \\
\hline \multicolumn{4}{|l|}{ Genetics education background } \\
\hline Taken $>1$ genetics course & 5.4 & 3.0 & 3.5 \\
\hline Taken 1 genetics course & 18.9 & 12.1 & 24.6 \\
\hline Learned some genetics in basic sciences & 73.0 & 81.8 & 68.4 \\
\hline Never learned about genetics & 2.7 & 3.0 & 3.5 \\
\hline Past education covered application of genetics in pharmacy & 2.7 & 9.1 & 12.3 \\
\hline
\end{tabular}

about genetics. For those that had some genetics education previously, very few $(3,9$, and $12 \%)$ reported that it included the application of genetics to pharmacy (Table 1). There were no significant differences seen in response to additional survey questions described below based on the amount of previous genetic education the students had completed.

Students' attitudes and beliefs toward pharmacogenomics were also evaluated (Table 2). When first asked if drug effects could be altered by genetic variability in patients, 96.2 and 99.5 agreed with this statement before and after taking the course, respectively. When asked whether "pharmacogenomics is relevant to patient care" and "pharmacogenomics can improve patient care," most students agreed in both the pre-survey $(95.7 \%)$ and the post-survey (98.4\%). In 2015 and 2016 students were also given a scenario in which their patient presented with intestinal bleeding after recently being prescribed Warfarin. Students were asked to respond by stating whether they agreed that as a pharmacist they would suggest ordering genetic tests to determine whether the warfarin treatment should be changed (Table 2). An increase in the number of students that agreed they would have genetic testing completed in the post-survey $(90.2 \%)$ was seen, as compared to the pre-survey (40.7\%). These findings suggest pharmacy students understand that pharmacogenomics may be helpful for inpatient care, but only after taking a course in pharmacogenomics do they understand the clinical applicability. Although students perceive that pharmacogenomics is relevant to and can improve patient care that does not mean that they necessarily hold themselves, as pharmacists, accountable to be knowledgeable in this area. To assess their perceptions of 
American Journal of Pharmaceutical Education 2018; 82 (9) Article 6424.

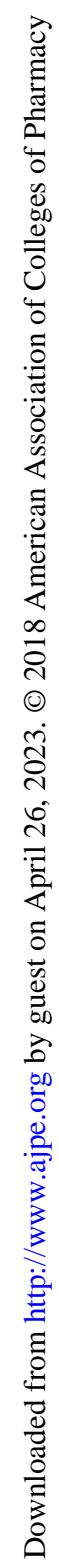

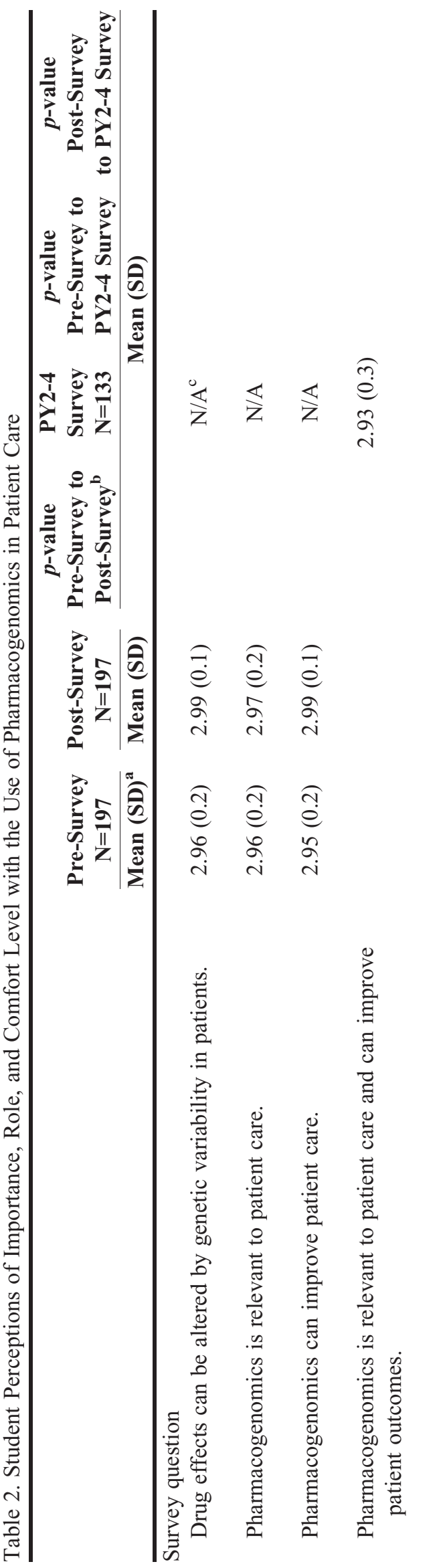

ลิ

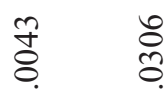

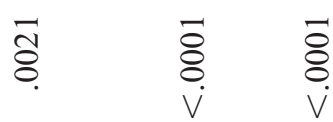

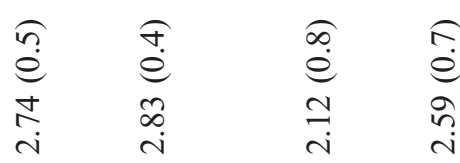

$\overrightarrow{8}$
$\dot{8}$

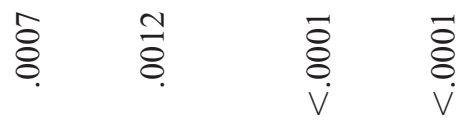

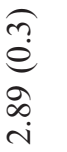

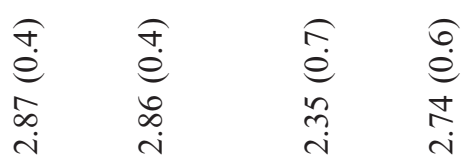

$\hat{e}$
$\stackrel{e}{e}$
in

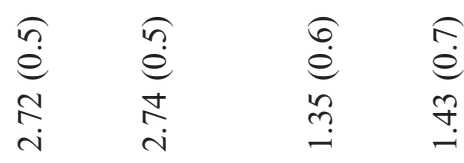

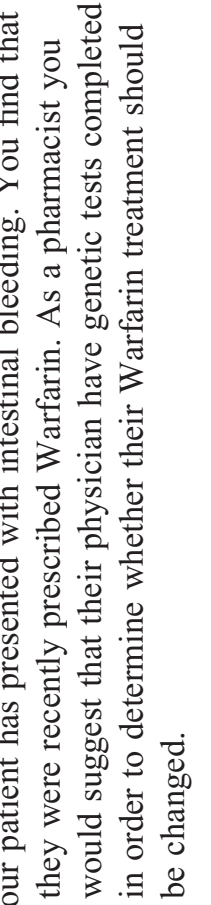

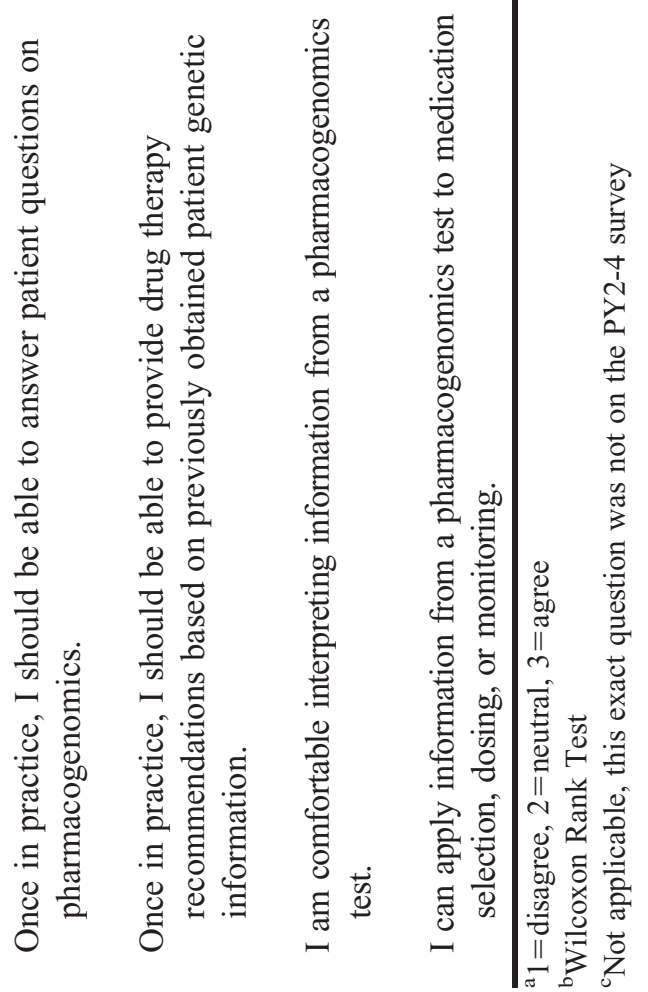




\section{American Journal of Pharmaceutical Education 2018; 82 (9) Article 6424.}

this aspect they were asked to respond to the following two statements "once in practice, I should be able to answer patient questions on pharmacogenomics" and "once in practice, I should be able to provide drug therapy recommendations based on previously obtained patient genetic information." While many students agreed to these statements prior to taking the course (74.2 and $74.6 \%$ ), there was a shift toward agreement that they should be involved in pharmacogenomics use once in practice, after they had completed the course (87.1 and $87.9 \%, p=.0007$ and $p=.0012$ ) (Table 2). Next, the authors wanted to determine whether student perception of their abilities to interpret and apply genetic information had changed once they completed the Principles in Genetics and Pharmacogenomics course. When assessing students' comfort level in interpreting information from a pharmacogenomics test, there was a significant increase in the number of students that agreed that they were comfortable after taking the course (52.7\%) as compared to before $(5.9 \%)$ (Table 2). Similarly, the number of students that agreed with the statement, "I can apply information from a pharmacogenomics test to medication selection, dosing, or monitoring" also increased substantially between the presurvey $(9.7 \%)$ and post-survey (80.1\%) (Table 2). These results indicate the utility of a pharmacogenomics course to adequately prepare pharmacy students to make clinical decisions linking genetics to therapeutic response.

The Principles of Genetics and Genomics course at WNE is during the spring of PY 1 year. Thus, the authors investigated whether students were encountering pharmacogenomics in other courses later in the curriculum, whether they observed its use in the pharmacy or clinic, and whether their perceptions changed as they approached graduation (Table 3). When second, third, and fourth year PharmD students were surveyed, $(84.2 \%$ responded that they had been taught pharmacogenomics at a memorable breadth and depth in at least one course since the PY1 Principles in Genetics and Pharmacogenomics course. Conversely, only $21.8 \%$ of students responded that they had observed pharmacogenomics used in pharmacy practice. Despite this lack of exposure in clinical settings, most students still agreed that the course was important to their education $(94.6 \%)$ and the field of pharmacogenomics is growing in pharmacy practice $(88.5 \%)$.

To determine if students' perceptions on the relevance of pharmacogenomics to patient care changed from their PY1 year, students were asked if they agreed with the statement, "pharmacogenomics is relevant to patient care and can improve patient outcomes." and almost all students still agreed (93\%) (Table 2). PY2-4 students were then asked if they agreed with the statements, "once in practice, I should be able to answer patient questions on pharmacogenomics" and "once in practice, I should be able to provide drug therapy recommendations based on previously obtained patient genetic information." Interestingly, agreement regarding answering patient questions returned nearly to baseline (77.7\%, 74.2\% PY1 Pre-Survey), while agreement regarding providing therapy recommendations remained significantly higher than prior to taking the Principles in Genetics and Pharmacogenomics course (85.3\%, $74.6 \%$ PY1 Pre-Survey) (Table 2). When questioned whether they were comfortable with interpreting genetic test results $(35.4 \%, 5.9 \% \mathrm{PY} 1$ pre-survey, $52.7 \% \mathrm{PY} 1$ postsurvey) or applying that information to medication therapy (69.8\%, 9.7\% PY1 Pre-survey, 80.1\% PY1 post-survey), PY2-4 students were less comfortable than they had been immediately upon completion of the course, but they were still significantly more comfortable in the application of pharmacogenomic information than they had been before taking the course (Table 2). Finally, the authors obtained a list of 18 required courses that occur later in the curriculum, which touch upon pharmacogenomics concepts (Table 4). The majority of these courses are integrated therapeutics modules ( $72.2 \%$ of required courses reported), which indicates that faculty are at least mentioning genetic factors

Table 3. Reintroduction and Use of Pharmacogenomics, and Perceptions of Importance of Pharmacogenomics to PY2-4 Students

\section{PY2-4 Responses}

$\mathrm{N}=\mathbf{1 3 3}$

Yes $(\%)$

84.2

Have you been taught pharmacogenomics at any level in any course since the genetics and genomics course?

Have you seen genetics/pharmacogenomics used in pharmacy practice?

21.8

78.2

Agree (\%)

94.6

This course was important to my pharmacy education. Pharmacogenomics is a growing field in the practice of pharmacy.
88.5
Disagree (\%) Disagree (\%)

$\begin{array}{rl}5.4 & 0.00 \\ 10.8 & 0.8\end{array}$




\section{American Journal of Pharmaceutical Education 2018; 82 (9) Article 6424.}

Table 4. List of Courses in Second, Third, and Fourth Years of the Curriculum that Touch upon Pharmacogenomic Principles as Reported by PY2-4 Students

\begin{tabular}{lc}
\hline & PY2-4 responses \\
Course Name & (N) 133) \\
\hline Principles of Pharmacokinetics & 39.06 \\
Principles of Pharmacology & 58.59 \\
Principles of Medicinal Chemistry & 41.41 \\
IPC\&PM Renal & 48.44 \\
IPC\&PM Respiratory & 46.09 \\
IPC\&PM Cardiovascular System I & 52.34 \\
IPC\&PM Cardiovascular System II & 49.22 \\
Applied Pharmacy Care I & 23.44 \\
IPC\&PM Gastrointestinal/Nutrition/Hepatic/Pancreatic & 58.14 \\
IPC\&PM Endocrine/Reproductive/Genitourinary & 54.65 \\
IPC\&PM Infectious Diseases I & 43.02 \\
IPC\&PM Infectious Diseases II & 47.67 \\
Applied Pharmacy Care II & 20.93 \\
IPC\&PM Dermatological/Musculoskeletal & 38.00 \\
IPC\&PM Neurological/Central Nervous System & 32.00 \\
IPC\&PM Psychological & 38.00 \\
IPC\&PM Hematological/Oncology & 56.00 \\
IPC\&PM Special Populations & 22.00 \\
Elective(s) & 26.56 \\
\hline Abbriation
\end{tabular}

Abbreviations: IPC = Integrated Patient Care. PM = Patient Management. IPC and PM courses are system/disease state based integrated pharmacology, medicinal chemistry, and therapeutics modules

as they are teaching about the use of specific therapies in various disease states.

\section{DISCUSSION}

In spite of the numerous advances in personalized medicine over the last decade, a knowledge gap in pharmacogenomics education and its use in clinical practice still exists. ${ }^{3}$ Having a required, stand-alone pharmacogenomics course, similar to ours at WNE may help to overcome this issue. The purpose of this study was to determine the influence of such a course on pharmacy student perception of their knowledge, as well as its clinical importance and their roles in utilizing genetic information in medication therapy. Furthermore, it aimed to determine whether students are exposed to pharmacogenomic principles later in the curriculum and how their perceptions changed, as they progressed through the Doctor of Pharmacy program. Based on student perceptions it appears the course is effective. Although students indicate that they have basic knowledge and understand the relevance of genetic testing and pharmacogenomics prior to the course, the course appears to provide them with specific understanding of pharmacogenomics in the clinical setting and their potential role as pharmacists in this field. This agrees with the findings of Moen and colleagues where $90 \%$ of pharmacy students agreed that pharmacogenomics could improve patient care, yet only $30 \%$ of respondents had taken a standalone course on the topic. Furthermore, approximately $70 \%$ of respondents in that study were not comfortable with their knowledge on the subject, which incidentally is the same percent that had not taken a pharmacogenomics course. ${ }^{8}$ Future studies may elucidate the role of recent pharmacy graduates that have had pharmacogenomics education in advocating for its use clinically and educating more senior practitioners on the benefits of personalized medicine.

It is worth noting that although the course described herein does appear to enhance the confidence of most students to interpret and apply genetic and pharmacogenomic information, there is still a small subset that do not feel comfortable in their ability to use genetic information as a pharmacist after the course. Due to the anonymous nature of the survey, responses could not be correlated with student performance. It is possible that students who performed poorly in the course are less comfortable in their knowledge and skills upon completion of the course. Thus, the results that were obtained may partly be due to inherent capabilities of the students. It is also reasonable to conclude that didactic coursework can only prepare the students to a certain degree, emphasizing the need for clinical, laboratory, and/or simulation types of coursework. In support of this, recent reports of courses using personal genetic testing, laboratory based elements (genotyping exercises of and/or by the students themselves), or simulation software 


\section{American Journal of Pharmaceutical Education 2018; 82 (9) Article 6424.}

indicate a better understanding and retention of information after the course. ${ }^{17-22}$

Due to the Principles in Genetics and Pharmacogenomics course at WNE taking place early in the curriculum, students have not completed any of the pharmacology or integrated therapeutics courses yet. Furthermore, while most respondents had learned some genetics in other basic science courses, they did not cover pharmacogenomics, and several students had not previously learned any genetics at all. To deal with this broad spectrum of prior knowledge, the course does provide basic genetic, pharmacological, and pharmaceutical principles, which are then linked to pharmacotherapy and pharmacogenomics. However, there is a concern that since students have not completed their therapeutics courses this may prevent their ability to fully grasp the clinical implications of pharmacogenomics. Importantly, it was found that many of the integrated therapeutics courses in the curriculum included pharmacogenomic concepts in their content. This curricular placement may provide a foundation of pharmacogenomics early on that can be built upon through upper level didactic coursework and potentially introductory and advanced pharmacy practice experiences (IPPEs and APPEs) as well.

This is supported by the results indicating that students maintain an understanding of their responsibility to use and interpret pharmacogenomics information to make therapeutic recommendations. It is likely that regular reiteration of pharmacogenomics throughout the curriculum has some influence in maintaining the knowledge and skills students acquired in their first year, especially considering the minimal exposure they have had in the pharmacy. This conclusion is in line with the recommendation from Vaksman and colleagues that a program offering pharmacogenomics coursework earlier in the curriculum and continuing to apply the concepts through constant reinforcement is most likely to cultivate and sustain student interest in the field and potentially increase the number of pharmacy practitioners with this expertise. ${ }^{9}$

There is always a concern of having enough time in a course to cover all necessary material. The most realistic scenario when it comes to the inclusion of pharmacogenomics in a course is to present relevant information as each drug is introduced. This could be completed in a similar manner to incorporation of new therapies, side effects, or resistance mechanisms in the course content, which may provide a more organic process than identifying large portions of material to remove to fit pharmacogenomics into non-standalone courses. Although the feasibility of using this process is likely to be greater if the curriculum includes a required pharmacogenomics course earlier in the curriculum as described here. Future research focusing on the depth and breadth of pharmacogenomics taught in these other courses may be useful in clarifying the time and effort that are required to incorporate pharmacogenomics into an established curriculum. For example, some disease states, such as oncology, are more likely to be treated with therapies where patient response is linked to genetics, potentially increasing the amount of time required to cover pharmacogenomics in those courses than in others.

The only student perception that increased significantly upon completion of the course, but then subsequently decreased back to baseline as students progressed through the curriculum was the concept that as practicing pharmacists they should be able to answer patient questions regarding pharmacogenomics. This may be explained by their responses to two other items, which indicated that they have not observed pharmacogenomics use in practice and that their pharmacy experience is primarily in retail settings. This is not surprising considering in a 2013 ASHP national survey of pharmacy practice in hospital settings, only $7 \%$ of respondent hospitals provide pharmacogenomic testing, which was actually an increase from $2.7 \%$ in 2009 and is conceivably higher than in most retail pharmacies. ${ }^{23} \mathrm{Stu}-$ dents are unlikely to have observed pharmacists answering patient questions on this topic, which may result in their altered perception of their role over time. As the use of personalized medicine becomes more common, it is anticipated that pharmacists in various settings will increasingly be required to counsel patients on pharmacogenomics.

Importantly, according to the ASHP Foundation Pharmacy Forecast for 2015-2019, it is likely that academic medical centers across the U.S. will begin implementing formal pharmacogenomics services for their patients within this timeframe. ${ }^{24}$ Pharmacogenomics implementation may also be moving in the direction of retail pharmacies, especially now that certain pharmacies sell kits for direct to consumer genetic analysis. In addition, a 2015 study among five community pharmacies found that the delivery of pharmacogenomic testing in a community pharmacy setting appears feasible, however pharmacist training and access to clinical support may be needed. ${ }^{25}$ Other studies are assessing the feasibility of adding pharmacogenomic testing to medication therapy management provided by pharmacists. ${ }^{26,27}$ It is likely that as clinical and retail settings begin to implement pharmacogenomic testing and students are being exposed to its merits in helping patients, there will be less uncertainty of their role in responding to questions regarding pharmacogenomics and its use. In support of this, as students progress through the program, they agree that this is an important part of their education and they believe that pharmacogenomics is a growing field within pharmacy practice.

There are several limitations to this study design that should be noted. For the surveys completed by PY1 


\section{American Journal of Pharmaceutical Education 2018; 82 (9) Article 6424.}

students, there could have been some bias as they received bonus credit for completing the surveys. This was done to increase the response rate, which was the case as compared to the surveys where respondents did not receive any compensation. To reduce the potential for bias, the participants who answered the surveys were anonymized. Another limitation of this study was that the range of the Likert scale was confined to three choices, whereas many studies use a 5-point scale. The reason for doing this was to limit the subjectivity that can occur with the use of qualifying adjectives used in 5-point scales. Furthermore, this study focused only on the perceptions of the students who had taken the Principles in Genetics and Pharmacogenomics course, not the outcomes or efficacy of the course per se. Finally, data from only one PharmD program housed in a private university were reported. It is possible that the results would vary if students from different pharmacy programs across the country were surveyed.

Future studies that assess the perception and knowledge of both IPPE/APPE preceptors and faculty across pharmacy programs in the US may be helpful to gain a clearer picture of the integration of pharmacogenomics throughout the didactic and experiential portions of curricula and determine their role in providing students with the knowledge and skills to use pharmacogenomics as future pharmacists. In addition, development sessions for faculty may help not only in providing basic knowledge of pharmacogenomics, but also provide insight into how to teach students about this topic. According to Murphy and colleagues in $2010,54.7 \%$ of pharmacy colleges had no plans for faculty development programs in pharmacogenomics. ${ }^{13}$ However, a 2012 study involving 58 faculty from 39 pharmacy schools demonstrated that as a result of participating in a train-the-trainer program in pharmacogenomics, faculty member participants gained confidence in teaching pharmacogenomics to their students, and the majority of participants indicated a high likelihood of adopting the program at their institution. ${ }^{7}$ Although there is no requirement for practicing pharmacists to complete continuing education on the subject of pharmacogenomics, it is a potential step to ensure that pharmacy professors and preceptors, who did not have instruction on this topic previously, have the knowledge to educate future pharmacists. ${ }^{6,28}$

\section{ACKNOWLEDGMENTS}

The authors would like to thank Dr. Evan Robinson and Dr. Christopher Hakala for advice during the development of this project. We would also like to thank Dr. Daniel Kennedy, Dr. Natalia Shcherbakova, and Dr. Shamima Khan for their constructive criticism of the manuscript. Finally, we must thank Dr. Quan Wei for her recommendations for statistical analysis of the data.

\section{REFERENCES}

1. Dickmann LJ, Ware JA. Pharmacogenomics in the age of personalized medicine. Drug Discov Today Technol. 2016;21-22:11-16. 2. Nelson MR, Johnson T, Warren L, et al. The genetics of drug efficacy: opportunities and challenges. Nat Rev Genet. 2016;17(4):197-206.

3. Rao US, Mayhew SL, Rao PS. Strategies for implementation of an effective pharmacogenomics program in pharmacy education.

Pharmacogenomics. 2015;16(8):905-911.

4. Roederer MW, Van Riper M, Valgus J, Knafl G, McLeod H. Knowledge, attitudes and education of pharmacists regarding pharmacogenetic testing. Personal Med. 2012;9(1):19-27.

5. McCullough KB, Formea CM, Berg KD, et al. Assessment of the pharmacogenomics educational needs of pharmacists. Am J Pharm Educ. 2011;75(3):Article 51.

6. Formea CM, Nicholson WT, McCullough KB, et al. Development and evaluation of a pharmacogenomics educational program for pharmacists. Am J Pharm Educ. 2013;77(1):Article 10.

7. Lee KC, Ma JD, Hudmon KS, Kuo GM. A train-the-trainer approach to a shared pharmacogenomics curriculum for US colleges and schools of pharmacy. Am J Pharm Educ. 2012;76(10):Article 193.

8. Moen M, Lamba J. Assessment of healthcare students' views on pharmacogenomics at the University of Minnesota.

Pharmacogenomics. 2012;13(13):1537-1545.

9. Vaksman N, Barnett M, Hakobyan L, Kutcher I, Louie MC. The impact of incorporating of pharmacogenomics into the pharmacy curriculum on student interest. Pharm Educ. 2012-11-01 2012;

12(1):31-36.

10. Johnson JA, Bootman JL, Evans WE, et al. Pharmacogenomics: a scientific revolution in pharmaceutical sciences and pharmacy practice. Report of the 2001-2002 Academic Affairs Committee. Am $J$ Pharm Educ. 2002;66:12S-15S.

11. Kisor DF, Smith HE, Grace E, Johnson SG, Weitzel KW, Farrell CL. The DNA of pharmacy education: CAPE outcomes and pharmacogenomics. 2015. https://www.aacp.org/sites/default/files/ 2017-10/The_DNA_of_Pharmacy_Education-CAPE_Outcomes_and_ Pharmacogenomics_2015.pdf. Accessed February 12, 2016.

12. American Society of Health-System Pharmacists. ASHP statement on the pharmacist's role in clinical pharmacogenomics. Am J Health-Syst Pharm. 2015;72(7):579-581.

13. Murphy JE, Green JS, Adams LA, Squire RB, Kuo GM, McKay A. Pharmacogenomics in the curricula of colleges and schools of pharmacy in the United States. Am J Pharm Educ. 2010;74(1):Article 7.

14. Feldenzer A, Aguilera MD, Sesto N, Spooner JJ, Kennedy DR. Mapping required biological science courses in pharmacy curricula to the PCOA exam. 117th Annual Meeting of the American Association of Colleges of Pharmacy, Anaheim, California, July 23-27, 2016. Am J Pharm Educ. 2016;80(5):Article S2.

15. Mrazek M, Koenig B, Skime M, et al. Assessing attitudes about genetic testing as a component of continuing medical education. Acad Psychiatry. 2007;31(6):447-451.

16. Faul F, Erdfelder E, Lang AG, Buchner A. G*Power 3: a flexible statistical power analysis program for the social, behavioral, and biomedical sciences. Behav Res Methods. 2007;39(2):175-191. 17. Adams SM, Anderson KB, Coons JC, et al. Advancing pharmacogenomics education in the core PharmD curriculum through student personal genomic testing. Am J Pharm Educ. 2016;80(1):Article 3.

18. Farrell CL, Pedigo NG, Messersmith AR. Application of genomic principles to pharmacotherapy of cancer. Am J Pharm Educ. 2014;78(3):Article 55. 


\section{American Journal of Pharmaceutical Education 2018; 82 (9) Article 6424.}

19. Knoell DL, Johnston JS, Bao S, Kelley KA. A genotyping exercise for pharmacogenetics in pharmacy practice. Am J Pharm Educ. 2009;73(3):Article 43.

20. Krynetskiy E, Calligaro IL. Introducing pharmacy students to pharmacogenomic analysis. Am J Pharm Educ. 2009;73(4):Article 71. 21. Springer JA, Iannotti NV, Kane MD, Haynes K, Sprague JE. Pharmacogenomics training using an instructional software system. Am J Pharm Educ. 2011;75(2):Article 32.

22. Weitzel KW, McDonough CW, Elsey AR, Burkley B, Cavallari LH, Johnson JA. Effects of using personal genotype data on student learning and attitudes in a pharmacogenomics course. Am J Pharm Educ. 2016;80(7):Article 122.

23. Pedersen CA, Schneider PJ, Scheckelhoff DJ. ASHP national survey of pharmacy practice in hospital settings: prescribing and transcribing-2013. Am J Health Syst Pharm. 2014;71(11):924-942. 24. Zellmer WA. Pharmacy forecast 2015-2019: strategic planning advice for pharmacy departments in hospitals and health systems.
2014. https://www.pharmacypracticeaccredit.org/resources/ pharmacy-forecast-2015-2019-strategic-planning-advice-forpharmacy-departments-in-hospitals-and-health-systems. Accessed January 3, 2017.

25. Moaddeb J, Mills R, Haga SB. Community pharmacists' experience with pharmacogenetic testing. J Am Pharm Assoc (2003). 2015;55(6):587-594.

26. Haga SB, Allen LaPointe NM, Moaddeb J, Mills R, Patel M, Kraus WE. Pilot study: incorporation of pharmacogenetic testing in medication therapy management services. Pharmacogenomics. 2014;15(14):1729-1737.

27. Reiss SM. American Pharmacists Association. Integrating pharmacogenomics into pharmacy practice via medication therapy management. J Am Pharm Assoc (2003). 2011;51(6):e64-e74. 28. Zembles T. An inservice program on pharmacogenetics to individualize drug therapy. Am J Pharm Educ. 2010;74(1): Article 10. 